\title{
Cardiovascular risk in patients with and without diabetes presenting with chronic coronary syndrome in 2004-2016
}

\author{
Esben Skov Jensen ${ }^{1}$, Kevin Kris Warnakula Olesen' ${ }^{1}$, Christine Gyldenkerne', Pernille Gro Thrane', \\ Lisette Okkels Jensen², Bent Raungaard ${ }^{3}$, Per Løgstrup Poulsen ${ }^{4}$, Reimar Wernich Thomsen ${ }^{5}$ and \\ Michael Maeng ${ }^{1 *}$ (D)
}

\begin{abstract}
Background: It was recently shown that new-onset diabetes patients without previous cardiovascular disease have experienced a markedly reduced risk of adverse cardiovascular events from 1996 to 2011. However, it remains unknown if similar improvements are present following the diagnosis of chronic coronary syndrome. The purpose of this study was to examine the change in cardiovascular risk among diabetes patients with chronic coronary syndrome from 2004 to 2016.

Methods: We included patients with documentation of coronary artery disease by coronary angiography between 2004 and 2016 in Western Denmark. Patients were stratified by year of index coronary angiography (2004-2006, 2007-2009, 2010-2012, and 2013-2016) and followed for two years. The main outcome was major adverse cardiovascular events (MACE) defined as myocardial infarction, ischemic stroke, or death. Analyses were performed separately in patients with and without diabetes. We estimated two-year risk of each outcome and adjusted incidence rate ratios (aIRR) using patients examined in 2004-2006 as reference.
\end{abstract}

Results: Among 5931 patients with diabetes, two-year MACE risks were 8.4\% in 2004-2006, 8.5\% in 2007-2009, and then decreased to 6.2\% in 2010-2012 and 6.7\% in 2013-2016 (2013-2016 vs 2004-2006: alRR 0.70, 95\% Cl 0.53-0.93). In comparison, 23,540 patients without diabetes had event rates of $6.3 \%, 5.2 \%, 4.2 \%$, and $3.9 \%$ for the study intervals (2013-2016 vs 2004-2006: alRR 0.57, 95\% Cl 0.48-0.68).

Conclusions: Between 2004 and 2016, the two-year relative risk of MACE decreased by 30\% in patients with diabetes and chronic coronary syndrome, but slightly larger absolute and relative reductions were observed in patients without diabetes.

Keywords: Diabetes, Coronary artery disease, Major adverse cardiovascular event, Trend

*Correspondence: michael.maeng@clin.au.dk

${ }^{1}$ Department of Cardiology, Aarhus University Hospital, Palle Juul-Jensens Boulevard 99, Aarhus N, Denmark

Full list of author information is available at the end of the article

\section{Background}

Among patients with diabetes, randomized clinical studies have shown that multifactorial medical intervention with tight regulation of blood glucose, blood pressure, and lipid-levels reduces the risk of myocardial infarction (MI) and premature death [1]. This subsequently led to changes of the diabetes guidelines with focus on prophylactic multifactorial intervention [2-6]. We recently 
found substantial reduction in the risk of MI among new-onset type 2 diabetes patients in Denmark without previous cardiovascular disease, simultaneous with the implementation of multifactorial intervention [7]. Further, following documentation of coronary artery disease $(\mathrm{CAD})$ in patients with diabetes, the management and treatment of CAD have also improved in the last decades with the documentation of coronary artery bypass grafting (CABG) being superior to percutaneous coronary intervention (PCI) when multivessel disease is present, the implementation of fractional flow reserve (FFR) measurement as an important diagnostic tool, and the development of newer-generation drug-eluting stents (DES) with lower risk of stent thrombosis being the most important improvements [8-10]. However, whether cardiovascular risk for diabetes patients with chronic coronary syndrome has changed over the last decades has not been examined in the setting of daily clinical practice on a nationwide level. Therefore, we investigated changes in cardiovascular risk among diabetes patients with chronic coronary syndrome from 2004 to 2016 and used patients without diabetes as a comparison cohort.
We hypothesized that substantial improvements in cardiovascular risk had taken place.

\section{Methods}

Data sources

The Western Denmark Heart Registry is a clinical database that provides prospective registration of all patients in Western Denmark undergoing cardiac intervention such as coronary angiography (CAG), PCI, and CABG since 1999 . The registry has previously been described in detail [11]. Using each patient's unique 10-digit identifier, patients can be linked with other national health care registries, including the Danish National Prescription Registry, the Civil Registration System, the Danish Register of Causes of Death, and the Danish National Patient Registry [12-15].

\section{Patient selection}

Patients undergoing CAG were identified using first-time procedures registered in the Western Denmark Heart Registry from 2004 through $2016(n=146,191)$ (Fig. 1). If a patient had multiple CAGs registered during this time,

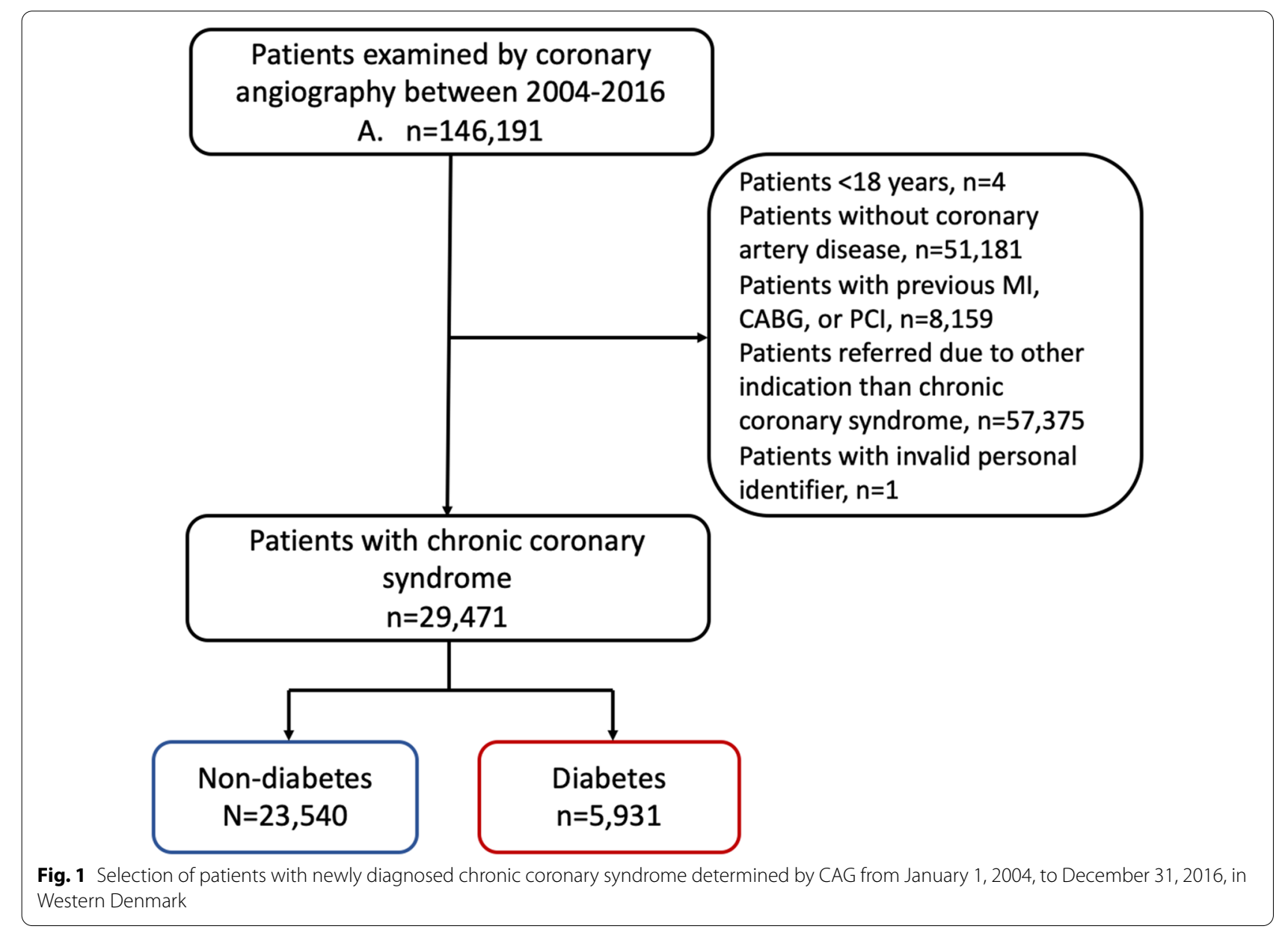


the first was considered the index examination. Four patients $<18$ years and 51,181 patients with no CAD were excluded from this analysis. Since we aimed to assess risk following the first-time diagnosis of chronic coronary syndrome by CAG, we excluded 8159 patients with previous $\mathrm{MI}, \mathrm{PCI}$, or CABG. Patients referred for CAG due to a different indication than chronic coronary syndrome were also excluded $(n=57,375)$.

\section{CAD}

Presence and extent of CAD were entered into the database by the interventional cardiologist immediately following examination. CAD was classified as either obstructive disease in 1,2 , or 3 vessels (with obstructive disease defined as $>50 \%$ diameter stenosis and FFR $\leq 0.80$ if measured) or as diffuse CAD defined as non-significant CAD involving $>1$ vessel. Patients with only a single stenosis $<50 \%$ or FFR $>0.80$ if measured were classified as no $C A D$ and excluded from the study.

\section{Diabetes}

Diabetes was defined as either (1) diet treatment only, non-insulin anti-diabetic treatment, or insulin ( \pm noninsulin anti-diabetic treatment) as registered in the Western Denmark Heart Registry, (2) diabetes diagnosis prior to CAG in the Danish National Patient Registry, or (3) collecting one or more prescriptions of insulin or non-insulin anti-diabetic treatment less than six months before CAG according to the Danish National Prescription Registry [12].

\section{Comorbidity}

Comorbidities were ascertained through the Danish National Patient Registry relying on diagnoses prior to CAG with full look-back (from 1977 and onwards). Information regarding smoking status, body mass index (BMI), and hypertension was ascertained through the Western Danish Heart Registry. We estimated burden of comorbidity using a modified Charlson's Comorbidity Index score, in which 'Diabetes, type I and II' and 'Diabetes with end-organ failure' were excluded in the final score [16].

\section{Medication}

Records of treatment with aspirin, adenosine diphosphate (ADP) receptor inhibitor, angiotensin-converting enzyme (ACE) inhibitor/angiotensin II receptor blocker (ARB), beta-blocker, and statin were collected from the Danish National Prescription Database. Medical treatment prior to CAG was defined as one or more redeemed prescriptions six months or less before CAG. Changes in medical treatment because of the CAG or peri-procedural diagnosis were investigated by looking at redeemed prescriptions six months or less after CAG in patients who completed six months of follow-up $(n=29,071)$ (Additional file 1: Tables S1 and S2).

\section{Outcomes}

The primary outcome was major adverse cardiovascular event (MACE); a composite of MI, ischemic stroke, and all-cause death. Secondary outcomes were the individual components of MACE, cardiac death, PCI, and CABG.

MI and ischemic stroke were identified in the Danish National Patient Registry [17, 18]. Vital status (alive, death, or emigration) was obtained through the Danish Civil Registration System [15]. Cardiac death included deaths resulting from ischemic heart disease, sudden cardiac death, heart failure, or sudden death, unspecified, according to death certificates from the Danish Register of Causes of Death [14].

Anatomical Therapeutic Chemical (ATC) codes used in the Danish Prescription Registry and International Classification of Diseases 10 (ICD-10) codes used in the Danish National Health Registry and the Danish Register of Causes of Death are listed in supplemental material of previous work [19].

\section{Statistical analysis}

Patients with chronic coronary syndrome were stratified by diabetes status at the time of examination and year of index CAG (2004-2006, 2007-2009, 2010-2012, and 2013-2016). We estimated two-year risks (cumulative incidence proportions) of MACE, MI, ischemic stroke, all-cause death, cardiac death, PCI, and CABG. Followup continued until an outcome event, death, emigration, or 24 months after CAG. Cumulative incidence proportion curves were constructed. We estimated the incidence rate ratio (IRR) using a modified Poisson regression with a robust variance-covariance estimator using the natural $\log$ of person-years as the offset [20]. IRRs were adjusted for sex, age, hypertension, previous ischemic stroke, peripheral artery disease, smoking, statin treatment, antiplatelet treatment, and oral anticoagulant treatment. Analyses of MACE, ischemic stroke, cardiac death, and all-cause death were additionally adjusted for atrial fibrillation and heart failure [21]. Patients examined between 2004 and 2006 were used as reference group throughout analyses.

We performed a number of sensitivity analyses. First, two-year MACE risks were compared between patients with and without diabetes (Additional file 1: Table S3). Secondly, we conducted a subgroup analysis of patients diagnosed with obstructive CAD at index CAG $(\mathrm{n}=23,858)$ (Additional file 1: Tables S4 and S5). Other analyses included stratifying by sex and age above or below 70 years (Additional file 1: Tables S6, S7, S8, and 
S9). Lastly, we performed an analysis of revascularization patterns as a consequence of the angiographic findings defined as PCI or CABG within three months after index CAG (Additional file 1: Table S10). Stata/MP 16.0 (StataCorp LLC, College Station, TX, USA) was used for all analyses.

\section{Results}

A total of 29,471 patients with chronic coronary syndrome, of whom 5931 (20\%) had diabetes, were included and eligible for analyses.

\section{Baseline characteristics}

Patient characteristics are outlined in Table 1 (diabetes) and Table 2 (non-diabetes). In general, similar changes in baseline characteristics were observed for diabetes and non-diabetes patients. The median age increased from 67 to 69 years for patients with diabetes and from 66 to 68 years for patients without diabetes from 2004-2006 to 2013-2016. We observed a reduction in the proportion of active smokers. Median BMI was 29 for patients with diabetes and 27 for patients without diabetes throughout the study period. Comorbidities increased in both groups with increasing Charlson's Comorbidity Index scores. The extent of CAD changed over time with a decrease in obstructive multivessel disease and increased presence of diffuse non-significant CAD.

Statin treatment after CAG was around $90 \%$ for all groups. The primary choice of statin changed from simvastatin to the more potent atorvastatin (Additional file 1: Tables S1 and S2) during the study period. For example, simvastatin and atorvastatin were used in $74 \%$ and $12 \%$ of patients with diabetes in 2004-2006, but these percentages had changed to $34 \%$ and $47 \%$ in $2013-2016$. The use of antihypertensive drugs also changed over time but in a more heterogenous way. The use of beta-blockers, ACE inhibitors, and thiazides decreased, the use of ARBs increased, and the use of calcium channel blockers remained stable. Finally, in the diabetes group, insulin treatment decreased from the first to the last study interval while use of non-insulin anti-diabetic medication increased.

\section{Clinical outcomes}

Tables 3 (diabetes) and 4 (non-diabetes) report the twoyear absolute and relative risks for the four study intervals and is graphically illustrated in Fig. 2. The risk of MACE decreased among patients with diabetes (8.4$6.7 \%$, adjusted incidence rate ratios (aIRR) $0.70,95 \% \mathrm{CI}$ $0.53-0.93)$ and patients without diabetes $(6.3-3.9 \%$, aIRR $0.57,95 \%$ CI $0.48-0.68$ ). The two-year risk of MACE remained around $2.5 \%$ higher among patients with diabetes in comparison to patients without diabetes through all study intervals (Additional file 1: Table S3). The results were consistent in both patients below and above 70 years (Additional file 1: Tables S8 and S9). Men had 1-2\% higher absolute risk of MACE compared to women in patients without diabetes, whereas sex differences was less pronounced among patients with diabetes (Additional file 1: Tables S6 and S7). Both men and women had reductions in MACE through the study period in accordance with our main analysis. In the diabetes group, the MACE reduction was primarily caused by halving the risk of ischemic stroke while relatively smaller, and statistically insignificant, reductions of MI and all-cause death were found. Similar results were found for patients without diabetes. These results were robust when we restricted the analyses to patients with obstructive CAD (i.e., excluding those with diffuse CAD) (Additional file 1: Tables S4 and S5).

\section{Revascularization}

The revascularization rates decreased within the first three months after index CAG for both PCI and CABG from the first to the last study interval (Table 5). This was found for both diabetes and non-diabetes patients. Similar results were found when analysing revascularization for the entire two-year study period (Additional file 1: Table S10). However, when restricting the analysis to patients with obstructive CAD, we found a change in the revascularization pattern with more patients being treated with PCI and fewer patients with CABG over time, a finding that was consistent among diabetes and non-diabetes patients (Additional file 1: Table S10).

\section{Discussion}

\section{Statement of principal findings}

Our main finding is that the two-year relative MACE risk decreased by $30 \%$ in patients with diabetes who presented with chronic coronary syndrome in Denmark from 2004 to 2016. This result was primarily caused by a reduction in ischemic stroke. However, since even larger relative and absolute risk reductions were observed among patients without diabetes, the gap between patients with and without diabetes did not change.

\section{Diabetes}

In the diabetes group, the absolute two-year risk of MACE decreased by $1.7 \%$ from 2004-2006 to $2013-$ 2016. This is likely the result of several guidelinedirected initiatives implemented in Denmark within the inclusion period. First, the focus on cardiovascular prevention has increased in diabetes patients where an intensified multifactorial intervention with tight regulation of blood glucose, blood pressure, and lipid-levels has proven to lower cardiovascular risk in diabetes 
Table 1 Baseline characteristics in patients with diabetes

\begin{tabular}{|c|c|c|c|c|c|c|c|c|}
\hline & $\begin{array}{l}2004-2006 \\
n=1066\end{array}$ & & $\begin{array}{l}2007-2009 \\
n=1507\end{array}$ & & $\begin{array}{l}2010-2012 \\
n=1523\end{array}$ & & $\begin{array}{l}2013-2016 \\
n=1835\end{array}$ & \\
\hline Median age, years (IQR) & $67(59-73)$ & & $67(60-74)$ & & $68(61-75)$ & & $69(61-75)$ & \\
\hline Male sex & 780 & 73.2 & 1061 & 70.4 & 1058 & 69.5 & 1301 & 70.9 \\
\hline Family history of ischemic heart disease & 486 & 45.6 & 671 & 44.5 & 697 & 45.8 & 795 & 43.3 \\
\hline Active smoker & 239 & 22.4 & 313 & 20.8 & 335 & 22.0 & 363 & 19.8 \\
\hline \multicolumn{9}{|l|}{ Comorbidity } \\
\hline Hypertension & 834 & 78.2 & 1272 & 84.4 & 1353 & 88.8 & 1583 & 86.3 \\
\hline Previous ischemic stroke & 37 & 3.5 & 52 & 3.5 & 60 & 3.9 & 75 & 4.1 \\
\hline Atrial fibrillation & 86 & 8.1 & 141 & 9.4 & 159 & 10.4 & 219 & 11.9 \\
\hline Peripheral artery disease & 101 & 9.5 & 162 & 10.7 & 167 & 11.0 & 184 & 10.0 \\
\hline Heart failure & 116 & 10.9 & 186 & 12.3 & 155 & 10.2 & 163 & 8.9 \\
\hline Renal disease & 32 & 3.0 & 51 & 3.4 & 67 & 4.4 & 92 & 5.0 \\
\hline Mean eGFR, mL/min (IQR) & $84(65-101)$ & & $88(68-107)$ & & $91(71-108)$ & & $90(71-108)$ & \\
\hline Median BMI, kg/m² (IQR) & $29(26-32)$ & & $29(26-33)$ & & $29(26-33)$ & & $29(26-33)$ & \\
\hline \multicolumn{9}{|l|}{ Modified Charlson Comorbidity Index score } \\
\hline 0 points & 853 & 80.0 & 1111 & 73.7 & 1111 & 72.9 & 1286 & 70.1 \\
\hline 1 point & 152 & 14.3 & 275 & 18.2 & 252 & 16.5 & 298 & 16.2 \\
\hline 2 point & 58 & 5.4 & 105 & 7.0 & 144 & 9.5 & 207 & 11.3 \\
\hline$\geq 3$ points & 3 & 0.3 & 16 & 1.1 & 16 & 1.1 & 44 & 2.4 \\
\hline \multicolumn{9}{|l|}{ CAD extent } \\
\hline $1 \mathrm{VD}$ & 307 & 28.8 & 450 & 29.9 & 502 & 33,0 & 570 & 31.1 \\
\hline $2 \mathrm{VD}$ & 269 & 25.2 & 336 & 22.3 & 312 & 20.5 & 400 & 21.8 \\
\hline $3 \mathrm{VD}$ & 438 & 41.1 & 456 & 30.3 & 348 & 22.8 & 393 & 21.4 \\
\hline Diffuse VD & 52 & 4.9 & 265 & 17.6 & 361 & 23.7 & 472 & 25.7 \\
\hline \multicolumn{9}{|l|}{ Medication } \\
\hline \multicolumn{9}{|l|}{ Statin } \\
\hline Before & 868 & 81.4 & 1244 & 82.5 & 1223 & 80.3 & 1441 & 78.5 \\
\hline After & 972 & 92.7 & 1349 & 91.4 & 1358 & 90.4 & 1598 & 88.6 \\
\hline \multicolumn{9}{|l|}{ Aspirin } \\
\hline Before & 876 & 82.2 & 1200 & 79.6 & 1162 & 76.3 & 1279 & 69.7 \\
\hline After & 941 & 89.7 & 1284 & 87.0 & 1285 & 85.5 & 1446 & 80.2 \\
\hline \multicolumn{9}{|l|}{ ADP-inhibitor } \\
\hline Before & 32 & 3.0 & 42 & 2.8 & 63 & 4.1 & 135 & 7.4 \\
\hline After & 519 & 49.5 & 655 & 44.4 & 651 & 43.3 & 763 & 42.3 \\
\hline \multicolumn{9}{|l|}{ Vitamin Kantagonists } \\
\hline Before & 91 & 8.5 & 126 & 8.4 & 131 & 8.6 & 144 & 7.8 \\
\hline After & 123 & 11.7 & 159 & 10.8 & 151 & 10.0 & 181 & 10.0 \\
\hline \multicolumn{9}{|l|}{ Non-vitamin Kantagonists } \\
\hline Before & 0 & 0.0 & 0 & 0.0 & 7 & 0.5 & 88 & 4.8 \\
\hline After & 0 & 0.0 & 0 & 0.0 & 14 & 0.9 & 107 & 5.9 \\
\hline \multicolumn{9}{|l|}{ Beta-blocker } \\
\hline Before & 732 & 68.7 & 894 & 59.3 & 820 & 53.8 & 867 & 47.2 \\
\hline After & 820 & 78.2 & 1050 & 71.1 & 1005 & 66.9 & 1084 & 60.1 \\
\hline \multicolumn{9}{|l|}{ ACE inhibitor } \\
\hline Before & 500 & 46.9 & 758 & 50.3 & 736 & 48.3 & 755 & 41.1 \\
\hline After & 540 & 51.5 & 791 & 53.6 & 733 & 48.8 & 727 & 40.3 \\
\hline \multicolumn{9}{|l|}{$A R B$} \\
\hline Before & 290 & 27.2 & 494 & 32.8 & 505 & 33.2 & 609 & 33.2 \\
\hline After & 302 & 28.8 & 490 & 33.2 & 499 & 33.2 & 613 & 34.0 \\
\hline Thiazides & & & & & & & & \\
\hline
\end{tabular}


Table 1 (continued)

\begin{tabular}{|c|c|c|c|c|c|c|c|c|}
\hline & $\begin{array}{l}2004-2006 \\
n=1066\end{array}$ & & $\begin{array}{l}2007-2009 \\
n=1507\end{array}$ & & $\begin{array}{l}2010-2012 \\
n=1523\end{array}$ & & $\begin{array}{l}2013-2016 \\
n=1835\end{array}$ & \\
\hline Before & 231 & 21.7 & 317 & 21.0 & 331 & 21.7 & 298 & 16.2 \\
\hline After & 247 & 23.5 & 309 & 20.9 & 317 & 21.1 & 281 & 15.6 \\
\hline \multicolumn{9}{|c|}{ Calcium channel blocker } \\
\hline Before & 423 & 39.7 & 621 & 41.2 & 662 & 43.5 & 705 & 38.4 \\
\hline After & 446 & 42.5 & 695 & 47.1 & 733 & 48.8 & 811 & 45.0 \\
\hline \multicolumn{9}{|l|}{ Insulin } \\
\hline Before & 349 & 32.7 & 495 & 32.8 & 503 & 33.0 & 547 & 29.8 \\
\hline After & 377 & 35.9 & 538 & 36.4 & 524 & 34.9 & 572 & 31.7 \\
\hline \multicolumn{9}{|c|}{ Non-insulin } \\
\hline Before & 644 & 60.4 & 936 & 62.1 & 1042 & 68.4 & 1346 & 73.4 \\
\hline After & 630 & 60.1 & 934 & 63.3 & 1035 & 68.9 & 1306 & 72.4 \\
\hline
\end{tabular}

Values are numbers and percentages unless otherwise stated

$A C E$ angiotensin converting enzyme, $A D P$ adenosine diphosphate, $A R B$ angiotensin-II receptor blocker, $B M I$ body mass index, $C A D$ coronary artery disease, eGFR estimated glomerular filtration rate, $I Q R$ inter-quartile range, $V D$ vessel disease

patients [1]. Although approximately 90\% of diabetes patients received statin treatment after the CAG, we observed a change in the primary choice of statin from simvastatin to the more potent atorvastatin during the study period, i.e., suggesting intensified lipid-lowering treatment [22]. Second, CABG is superior to PCI in patients with diabetes plus obstructive multivessel disease $[10,23]$. In the diabetes cohort, the use of PCI was reduced by an absolute $8 \%$ while CABG decreased from 23\% in 2004-2006 and remained stable around $18 \%$ throughout the last three study intervals. This suggests adherence to clinical guidelines in a time where FFR often led to downgrading of multivessel disease and where PCI in general tended to be preferred over CABG. Third, newer-generation DES have replaced bare-metal stents and first-generation DES during the study period. Newer-generation DES reduce MACE rates up to five years after PCI compared with first-generation DES [9, 24] and the two-year follow-up period may be too short to capture the benefit of newer-generation DES. Newergeneration DES also displayed higher safety in patients with diabetes $[25,26]$. However, the main reduction among diabetes patients was caused by reduced risk of ischemic stroke while patients without diabetes had reduced risk of all cardiovascular events.

\section{Obstructive CAD}

Fewer patients were classified as having obstructive multivessel CAD while more were classified as diffuse non-obstructive CAD. Theoretically, this can be explained by earlier detection of CAD (lead time bias), delayed progression of $\mathrm{CAD}$, or changed perception of CAD significance. Since the median age increased from
66 years in 2004-2006 to 68 years in 2013-2016, we find it unlikely that lead time bias and delayed progression of CAD are the main explanations for the observed reduced rates of multi-vessel disease. In contrast, the gradual implementation of intracoronary physiology measurements, such as FFR, to assist visual assessment of intermediate stenoses has undoubtedly led to downgrading of CAD severity since visual assessment alone tend to overestimate disease significance [27]. Importantly, MACE also decreased when we restricted our analyses to only include patients with obstructive CAD, i.e., the reduction of events was not explained by inclusion of more patients with diffuse CAD due to a changed registration pattern of non-obstructive CAD. Furthermore, the reduced cardiovascular risk among patients with obstructive CAD is presumably an underestimation of the actual reduced risk as we expect that some of the patients with "obstructive" CAD in the earlier study periods would have been classified as non-obstructive in the later periods when FFR became a standard tool in our daily clinical practice. Finally, in our sensitivity analysis of patients with obstructive CAD, it is noteworthy that the "downgrading" of CAD severity led to more use of PCI and less use of CABG among both diabetes and non-diabetes patients.

\section{Comparison with other work}

We have not been able to identify previous studies looking at changes in cardiovascular outcomes among patients with diabetes and chronic coronary syndrome. Our results, however, are in accordance with our previous study looking at improvements in 7-years outcomes among Danish patients with new-onset diabetes from 
Table 2 Baseline characteristics in patients without diabetes

\begin{tabular}{|c|c|c|c|c|c|c|c|c|}
\hline & $\begin{array}{l}2004-2006 \\
n=4847\end{array}$ & & $\begin{array}{l}2007-2009 \\
n=6104\end{array}$ & & $\begin{array}{l}2010-2012 \\
\mathrm{n}=5547\end{array}$ & & $\begin{array}{l}2013-2016 \\
n=7042\end{array}$ & \\
\hline Mean age, years (IQR) & $66(58-74)$ & & $67(59-74)$ & & $67(59-75)$ & & $68(59-75)$ & \\
\hline Male sex & 3554 & 73.3 & 4279 & 70.1 & 3732 & 67.3 & 4843 & 68.8 \\
\hline Family history & 2290 & 47.2 & 2892 & 47.4 & 2687 & 48.4 & 3218 & 45.7 \\
\hline Active smoker & 1293 & 26.7 & 1401 & 23.0 & 1275 & 23.0 & 1467 & 20.8 \\
\hline \multicolumn{9}{|l|}{ Comorbidity } \\
\hline Hypertension & 2875 & 59.3 & 3949 & 64.7 & 3736 & 67.4 & 4488 & 63.7 \\
\hline Previous ischemic stroke & 42 & 0.9 & 88 & 1.4 & 113 & 2.0 & 158 & 2.2 \\
\hline Atrial fibrillation & 391 & 8.1 & 499 & 8.2 & 474 & 8.5 & 643 & 9.1 \\
\hline Peripheral artery disease & 260 & 5.4 & 361 & 5.9 & 363 & 6.5 & 427 & 6.1 \\
\hline Heart failure & 390 & 8.0 & 434 & 7.1 & 357 & 6.4 & 358 & 5.1 \\
\hline Renal disease & 61 & 1.3 & 93 & 1.5 & 91 & 1.6 & 171 & 2.4 \\
\hline Mean eGFR, mL/min (IQR) & $81(66-96)$ & & $86(70-102)$ & & $89(74-104)$ & & $89(74-104)$ & \\
\hline Median BMI, kg/m² (IQR) & $27(24-29)$ & & $27(24-30)$ & & $27(24-29)$ & & $27(24-29)$ & \\
\hline \multicolumn{9}{|c|}{$\begin{array}{l}\text { Modified Charlson Comorbidity } \\
\text { Index score }\end{array}$} \\
\hline 0 points & 3931 & 81.1 & 4568 & 74.8 & 3917 & 70.6 & 4799 & 68.1 \\
\hline 1 point & 607 & 12.5 & 929 & 15.2 & 893 & 16.1 & 1162 & 16.5 \\
\hline 2 point & 222 & 4.6 & 411 & 6.7 & 488 & 8.8 & 696 & 9.9 \\
\hline$\geq 3$ points & 87 & 1.8 & 196 & 3.2 & 249 & 4.5 & 385 & 5.5 \\
\hline \multicolumn{9}{|l|}{ CAD extent } \\
\hline $1 \mathrm{VD}$ & 1764 & 36.4 & 2321 & 38.0 & 2049 & 36.9 & 2661 & 37.8 \\
\hline $2 \mathrm{VD}$ & 1236 & 25.5 & 1437 & 23.5 & 1141 & 20.6 & 1391 & 19.8 \\
\hline $3 \mathrm{VD}$ & 1530 & 31.6 & 1386 & 22.7 & 1029 & 18.6 & 1132 & 16.1 \\
\hline Diffuse VD & 317 & 6.5 & 960 & 15.7 & 1328 & 23.9 & 1858 & 26.4 \\
\hline \multicolumn{9}{|l|}{ Medication } \\
\hline \multicolumn{9}{|l|}{ Statin } \\
\hline Before & 3141 & 64.8 & 4136 & 67.8 & 3667 & 66.1 & 4490 & 63.8 \\
\hline After & 4347 & 91.4 & 5453 & 90.9 & 4879 & 89.1 & 6195 & 89.0 \\
\hline \multicolumn{9}{|l|}{ Aspirin } \\
\hline Before & 3795 & 78.3 & 4621 & 75.7 & 4009 & 72.3 & 4717 & 67.0 \\
\hline After & 4105 & 86.3 & 5067 & 84.5 & 4555 & 83.2 & 5500 & 79.0 \\
\hline \multicolumn{9}{|l|}{ ADP-inhibitor } \\
\hline Before & 148 & 3.1 & 145 & 2.4 & 215 & 3.9 & 487 & 6.9 \\
\hline After & 2540 & 53.4 & 2962 & 49.4 & 2535 & 46.3 & 3240 & 46.5 \\
\hline \multicolumn{9}{|l|}{ Vitamin Kantagonists } \\
\hline Before & 354 & 7.3 & 427 & 7.0 & 360 & 6.5 & 376 & 5.3 \\
\hline After & 519 & 10.7 & 535 & 8.9 & 471 & 8.6 & 484 & 7.0 \\
\hline \multicolumn{9}{|l|}{ Non-vitamin Kantagonists } \\
\hline Before & 0 & 0.0 & $<5$ & 0.0 & 38 & 0.7 & 268 & 3.8 \\
\hline After & 0 & 0.0 & $<5$ & 0.0 & 61 & 1.1 & 347 & 5.0 \\
\hline \multicolumn{9}{|l|}{ Beta-blocker } \\
\hline Before & 3348 & 69.1 & 3674 & 60.2 & 2868 & 51.7 & 2756 & 39.1 \\
\hline After & 3582 & 75.3 & 4219 & 70.3 & 3498 & 63.9 & 3707 & 53.2 \\
\hline \multicolumn{9}{|l|}{ ACE inhibitor } \\
\hline Before & 1260 & 26.0 & 1643 & 26.9 & 1587 & 28.6 & 1590 & 22.6 \\
\hline After & 1534 & 32.3 & 1947 & 32.5 & 1749 & 31.9 & 1694 & 24.3 \\
\hline \multicolumn{9}{|l|}{$A R B$} \\
\hline Before & 667 & 13.8 & 976 & 16.0 & 978 & 17.6 & 1479 & 21.0 \\
\hline After & 731 & 15.4 & 1041 & 17.4 & 1057 & 19.3 & 1564 & 22.5 \\
\hline
\end{tabular}


Table 2 (continued)

\begin{tabular}{|c|c|c|c|c|c|c|c|c|}
\hline & $\begin{array}{l}2004-2006 \\
n=4847\end{array}$ & & $\begin{array}{l}2007-2009 \\
n=6104\end{array}$ & & $\begin{array}{l}2010-2012 \\
\mathrm{n}=5547\end{array}$ & & $\begin{array}{l}2013-2016 \\
n=7042\end{array}$ & \\
\hline \multicolumn{9}{|c|}{ Thiazides } \\
\hline Before & 891 & 18.4 & 1077 & 17.6 & 954 & 17.2 & 917 & 13.0 \\
\hline After & 964 & 20.3 & 1111 & 18.5 & 930 & 17.0 & 914 & 13.1 \\
\hline \multicolumn{9}{|c|}{ Calcium channel blocker } \\
\hline Before & 1601 & 33.0 & 1958 & 32.1 & 1741 & 31.4 & 1982 & 28.1 \\
\hline After & 1777 & 37.4 & 2408 & 40.1 & 2211 & 40.4 & 2550 & 36.6 \\
\hline
\end{tabular}

Values are numbers and percentages unless otherwise stated. To preserve patient anonymity following Danish data regulations, cells with $<5$ observations are presented as such

$A C E$ angiotensin converting enzyme, $A D P$ adenosine diphosphate, $A R B$ angiotensin-Il receptor blocker, $B M I$ body mass index, $C A D$ coronary artery disease, $e G F R$ estimated glomerular filtration rate, $I Q R$ inter-quartile range, $V D$ vessel disease

Table 3 Two-year risk of adverse cardiovascular outcomes after coronary angiography in elective diabetes patients with chronic coronary syndrome

\begin{tabular}{|c|c|c|c|c|c|}
\hline & Patients & Events & $\begin{array}{l}\text { Two-year cumultive } \\
\text { incidence proportion } \\
(95 \% \mathrm{Cl})\end{array}$ & Unadjusted IRR (95\% Cl) & Adjusted IRR* $(95 \% \mathrm{Cl})$ \\
\hline \multicolumn{6}{|l|}{ MACE } \\
\hline 2004-2006 & 1066 & 89 & $8.4 \%(6.9-10.3)$ & Reference & Reference \\
\hline $2007-2009$ & 1507 & 126 & $8.5 \%(7.2-10.0)$ & $1.01(0.77-1.33)$ & $0.96(0.73-1.27)$ \\
\hline 2010-2012 & 1523 & 94 & $6.2 \%(5.2-7.6)$ & $0.73(0.55-1.98)$ & $0.67(0.50-0.91)$ \\
\hline $2013-2016$ & 1835 & 121 & $6.7 \%(5.6-7.9)$ & $0.78(0.59-1.03)$ & $0.70(0.53-0.93)$ \\
\hline \multicolumn{6}{|c|}{ Myocardial infarction } \\
\hline 2004-2006 & 1066 & 42 & $4.0 \%(3.0-5.4)$ & Reference & Reference \\
\hline $2007-2009$ & 1507 & 57 & $3.9 \%(3.0-5.0)$ & $0.97(0.65-1.45)$ & $0.96(0.64-1.43)$ \\
\hline 2010-2012 & 1523 & 61 & $4.1 \%(3.2-5.2)$ & $1.02(0.69-1.51)$ & $0.97(0.65-1.44)$ \\
\hline 2013-2016 & 1835 & 66 & $3.7 \%(2.9-4.7)$ & $0.91(0.61-1.34)$ & $0.85(0.57-1.25)$ \\
\hline \multicolumn{6}{|c|}{ Ischemic stroke } \\
\hline 2004-2006 & 1066 & 36 & $3.4 \%(2.5-4.7)$ & Reference & Reference \\
\hline $2007-2009$ & 1507 & 40 & $2.7 \%(2.0-3.7)$ & $0.79(0.50-1.24)$ & $0.76(0.48-1.21)$ \\
\hline 2010-2012 & 1523 & 24 & $1.6 \%(1.1-2.4)$ & $0.46(0.27-0.78)$ & $0.40(0.24-0.69)$ \\
\hline 2013-2016 & 1835 & 35 & $2.0 \%(1.4-2.7)$ & $0.56(0.35-0.89)$ & $0.47(0.29-0.76)$ \\
\hline \multicolumn{6}{|c|}{ Cardiac death } \\
\hline 2004-2006 & 1066 & 24 & $2.3 \%(1.5-3.4)$ & Reference & Reference \\
\hline 2007-2009 & 1507 & 41 & $2.8 \%(2.1-3.8)$ & $1.22(0.74-2.20)$ & $1.12(0.67-1.87)$ \\
\hline 2010-2012 & 1523 & 17 & $1.1 \%(0.7-1.8)$ & $0.50(0.27-0.92)$ & $0.45(0.24-0.85)$ \\
\hline 2013-2016 & 1835 & 30 & $1.7 \%(1.2-2.4)$ & $0.73(0.42-1.24)$ & $0.65(0.38-1.14)$ \\
\hline \multicolumn{6}{|l|}{ Death } \\
\hline 2004-2006 & 1066 & 64 & $6.0 \%(4.7-7.6)$ & Reference & Reference \\
\hline 2007-2009 & 1507 & 115 & $7.6 \%$ (6.4-9.1) & $1.28(0.95-1.74)$ & $1.21(0.89-1.64)$ \\
\hline 2010-2012 & 1523 & 85 & $5.6 \%(4.5-6.9)$ & $0.93(0.67-1.28)$ & $0.84(0.61-1.17)$ \\
\hline $2013-2016$ & 1835 & 97 & $5.3 \%(4.4-6.4)$ & $0.88(0.64-1.21)$ & $0.78(0.56-1.06)$ \\
\hline
\end{tabular}

*Adjusted for sex, age, smoking, hypertension, previous ischemic stroke, peripheral artery disease, statin treatment, antiplatelet treatment, and oral anti-coagulant treatment. Ischemic stroke and death were additionally adjusted for atrial fibrillation and heart failure

1996 to 2011 [28] as well as a Swedish study examining outcomes among patients with prevalent diabetes from 1998 to 2014 [29]. Moreover, two Swedish studies compared outcomes for all patients with acute coronary syndrome from 1995 to 2014 [30, 31] but differed concerning inclusion criteria (chronic vs acute coronary syndrome), study period, and lack of stratification based on presence of diabetes. Still, the studies share 
Table 4 Two-year risk of adverse cardiovascular outcomes after coronary angiography in elective non-diabetes patients with chronic coronary syndrome

\begin{tabular}{|c|c|c|c|c|c|}
\hline & Patients & Events & $\begin{array}{l}\text { Two-year cumulative } \\
\text { incidence proportion } \\
(95 \% \mathrm{Cl})\end{array}$ & Unadjusted IRR (95\% CI) & Adjusted IRR* $(95 \% \mathrm{Cl})$ \\
\hline \multicolumn{6}{|l|}{ MACE } \\
\hline 2004-2006 & 4847 & 302 & $6.3 \%(5.6-7.0)$ & Reference & Reference \\
\hline $2007-2009$ & 6104 & 312 & $5.2 \%(4.6-5.7)$ & $0.81(0.69-0.96)$ & $0.81(0.69-0.95)$ \\
\hline 2010-2012 & 5547 & 243 & $4.2 \%(3.9-5.0)$ & $0.69(0.59-0.82)$ & $0.65(0.55-0.78)$ \\
\hline 2013-2016 & 7042 & 272 & $3.9 \%(3.5-4.4)$ & $0.61(0.52-0.72)$ & $0.57(0.48-0.68)$ \\
\hline \multicolumn{6}{|c|}{ Myocardial infarction } \\
\hline 2004-2006 & 4847 & 154 & $3.2 \%(2.8-3.8)$ & Reference & Reference \\
\hline $2007-2009$ & 6104 & 173 & $2.9 \%(2.5-3.3)$ & $0.89(0.71-1.10)$ & $0.89(0.72-1.11)$ \\
\hline 2010-2012 & 5547 & 125 & $2.3 \%(1.0-2.7)$ & $0.70(0.55-0.89)$ & $0.68(0.54-0.87)$ \\
\hline 2013-2016 & 7042 & 175 & $2.5 \%(2.2-2.9)$ & $0.77(0.62-0.96)$ & $0.75(0.60-0.93)$ \\
\hline \multicolumn{6}{|c|}{ Ischemic stroke } \\
\hline 2004-2006 & 4847 & 72 & $1.5 \%(1.2-1.9)$ & Reference & Reference \\
\hline 2007-2009 & 6104 & 69 & $1.2 \%(0.9-1.5)$ & $0.76(0.54-1.05)$ & $0.71(0.51-0.99)$ \\
\hline 2010-2012 & 5547 & 64 & $1.2 \%(0.9-1.5)$ & $0.77(0.55-1.07)$ & $0.65(0.46-0.92)$ \\
\hline 2013-2016 & 7042 & 62 & $0.9 \%(0.7-1.1)$ & $0.58(0.42-0.82)$ & $0.48(0.34-0.68)$ \\
\hline \multicolumn{6}{|c|}{ Cardiac death } \\
\hline $2004-2006$ & 4847 & 102 & $2.1 \%(1.8-2.6)$ & Reference & Reference \\
\hline 2007-2009 & 6104 & 99 & $1.6 \%(1.4-2.0)$ & $0.77(0.58-1.01)$ & $0.77(0.58-1.87)$ \\
\hline 2010-2012 & 5547 & 65 & $1.2 \%(0.9-1.5)$ & $0.55(0.40-0.76)$ & $0.52(0.38-0.71)$ \\
\hline 2013-2016 & 7042 & 50 & $0.7 \%(0.5-0.9)$ & $0.33(0.24-0.47)$ & $0.31(0.22-0.44)$ \\
\hline \multicolumn{6}{|l|}{ Death } \\
\hline 2004-2006 & 4847 & 229 & $4.7 \%(4.2-5.4)$ & Reference & Reference \\
\hline 2007-2009 & 6104 & 270 & $4.4 \%(3.9-5.0)$ & $0.93(0.78-1.11)$ & $0.95(0.79-1.13)$ \\
\hline 2010-2012 & 5547 & 219 & $4.0 \%(3.5-4.5)$ & $0.83(0.69-1.00)$ & $0.80(0.66-0.97)$ \\
\hline 2013-2016 & 7042 & 216 & $3.1 \%(2.7-3.5)$ & $0.64(0.53-0.77)$ & $0.61(0.51-0.74)$ \\
\hline
\end{tabular}

*Adjusted for sex, age, smoking, hypertension, previous ischemic stroke, peripheral artery disease, statin treatment, antiplatelet treatment, and oral anti-coagulant treatment. Ischemic stroke and death were additionally adjusted for atrial fibrillation and heart failure

similarities by including a Scandinavian cohort treated in a national, tax-payer funded, public health care system, and the overall trends with reduced cardiovascular risk over the study period.

\section{Clinical implications}

It was recently shown that the risk of adverse cardiovascular events among patients with new-onset diabetes without previous cardiovascular disease decreased markedly from 1996 to 2011, drawing close to the cardiovascular risk of patients without diabetes [28]. In our study, we found that the relative risk of MACE decreased by $30 \%$ in patients with diabetes from 2004-2016, although their risk remained substantially increased compared to patients without diabetes. Therefore, an early and aggressive treatment strategy (i.e. cholesterol lowering drugs, blood pressure management, exercise, diet counseling, and smoking cessation) before the development of cardiovascular disease seems essential in order to minimize cardiovascular risk among diabetes patients, and such a multifactorial strategy, as documented by fewer active smokers and more use of high-intensity statins, likely played a role for the $30 \%$ risk reduction observed among the diabetes patients.

\section{Limitations}

Our study has some limitations to consider. The definition of MI was revised in 2007 and again in 2012 following the introduction of new high-sensitive cardiac troponin assays $[32,33]$. Lowering of the $99^{\text {th }}$ percentile upper normal reference limit due to improved biomarker sensitivity enabled smaller increases in troponin levels to meet the MI criteria. The lower MI diagnosis threshold in the later examination year intervals may underestimate the true reduction in MI during the study period [34].

Due to lack of biochemical data on our study group, we were unable to differentiate between prediabetic patients and normoglycemic patients in the non-diabetes group 


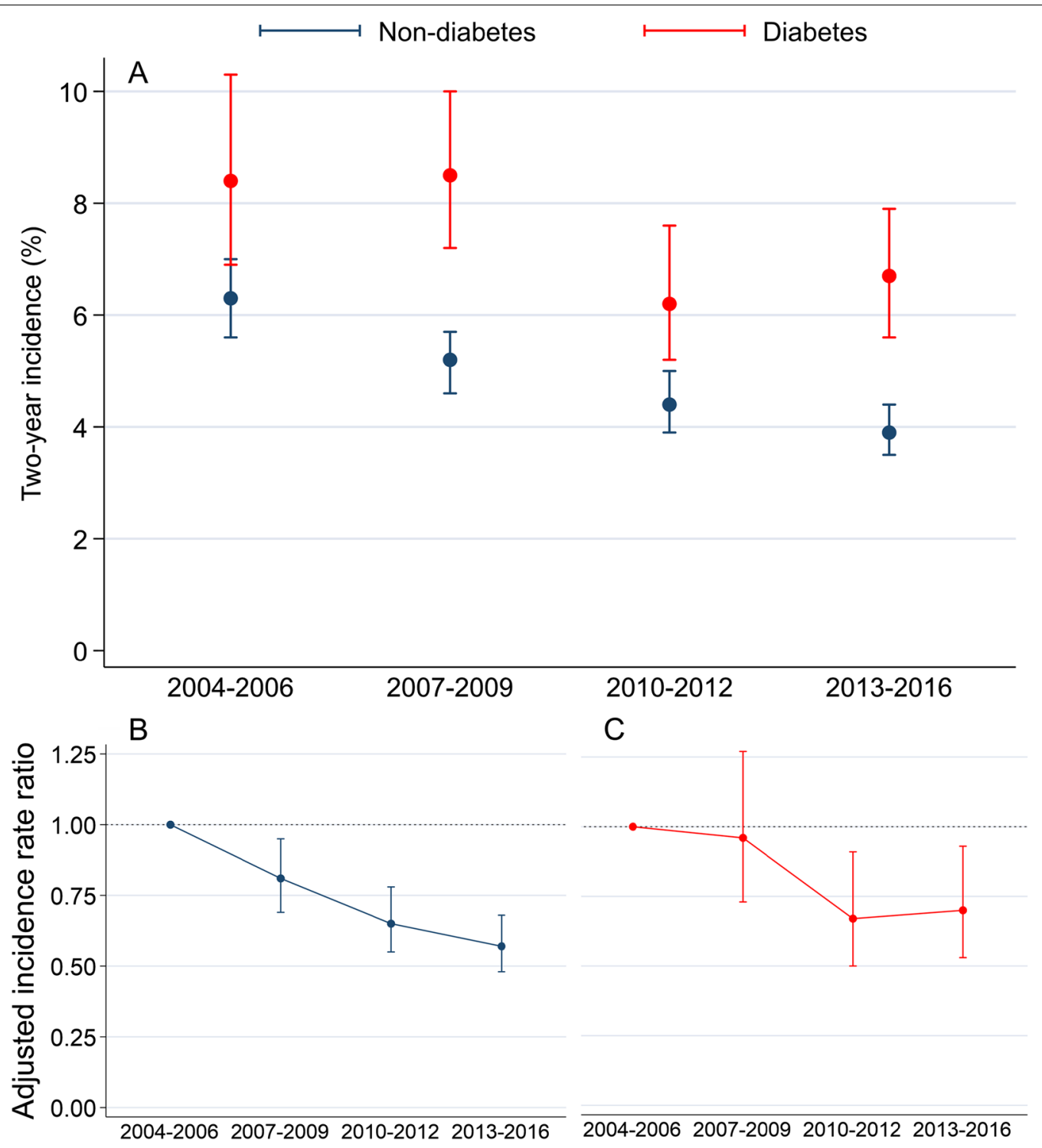

Fig. 2 Two-year risks of MACE with 95\% confidence intervals (a) and adjusted IRR in non-diabetes (b) and in diabetes (c) patients from 2004 to 2016

and investigate potential differences in cardiovascular outcomes [35, 36].

It is difficult to distinguish between type 1 and 2 diabetes based on registries alone. However, type 2 diabetes is by far the most common diabetes type in this age group and our results are thus mainly representative of patients with type 2 diabetes. As such, our results may not be representative for type 1 diabetes patients.

All studies assessing changes over time are limited by the fact that multiple changes have taken place during a long study period. While the main finding is that a large relative risk reduction was observed, which thereby shows that cardiovascular risk reduction is possible even in a 12-year period, it is difficult to define a specific cause.

Finally, our results were obtained in a tax-payer funded, public health care system with equal access for all citizens, and the external validity to societies with greater socioeconomic disparities needs confirmation.

\section{Conclusion}

In Denmark from 2004 to 2016, we found a reduced twoyear risk of MACE among both diabetes and non-diabetes patients with chronic coronary syndrome. However, despite improvements in cardiovascular risk and changed 
Table 5 Coronary revascularization among diabetes and non-diabetes patients within three months after index coronary angiography

\section{Patients Events 3-month cumulative incidence proportions \\ Unadjusted IRR Adjusted IRR* $(95 \% \mathrm{Cl})$} $(95 \% \mathrm{Cl})$

\begin{tabular}{|c|c|c|c|c|c|}
\hline \multicolumn{6}{|l|}{ Diabetes } \\
\hline \multicolumn{6}{|c|}{ Percutaneous coronary intervention } \\
\hline 2004-2006 & 1066 & 494 & $46.3 \%(43.4-49.3)$ & Reference & Reference \\
\hline $2007-2009$ & 1507 & 598 & $39.7 \%(37.2-42.2)$ & $0.76(0.65-0.89)$ & $0.76(0.65-0.90)$ \\
\hline 2010-2012 & 1523 & 603 & $39.6 \%(37.1-42.1)$ & $0.76(0.65-0.89)$ & $0.76(0.65-0.89)$ \\
\hline $2013-2016$ & 1835 & 696 & $37.9 \%(35.7-40.2)$ & $0.71(0.61-0.82)$ & $0.70(0.60-0.82)$ \\
\hline \multicolumn{6}{|c|}{ Coronary artery bypass grafting } \\
\hline 2004-2006 & 1066 & 240 & $22.5 \%(20.0-25.0)$ & Reference & Reference \\
\hline 2007-2009 & 1507 & 273 & $18.1 \%(16.2-20.1)$ & $0.76(0.63-0.92)$ & $0.76(0.62-0.92)$ \\
\hline 2010-2012 & 1523 & 245 & $16.1 \%(14.2-17.9)$ & $0.66(0.54-0.80)$ & $0.65(0.53-0.80)$ \\
\hline 2013-2016 & 1835 & 342 & $18.6 \%(16.9-20.4)$ & $0.79(0.65-0.95)$ & $0.80(0.66-0.96)$ \\
\hline \multicolumn{6}{|l|}{ Non-diabetes } \\
\hline \multicolumn{6}{|c|}{ Percutaneous coronary intervention } \\
\hline 2004-2006 & 4847 & 2403 & $49.6 \%(48.2-51.0)$ & Reference & Reference \\
\hline 2007-2009 & 6104 & 2766 & $45.3 \%(44.1-46.6)$ & $0.84(0.78-0.91)$ & $0.85(0.79-0.92)$ \\
\hline 2010-2012 & 5547 & 2396 & $43.2 \%(41.9-44.5)$ & $0.77(0.72-0.84)$ & $0.79(0.73-0.85)$ \\
\hline 2013-2016 & 7042 & 2887 & $41.0 \%(39.9-42.2)$ & $0.71(0.66-0.76)$ & $0.72(0.67-0.78)$ \\
\hline \multicolumn{6}{|c|}{ Coronary artery bypass grafting } \\
\hline 2004-2006 & 4847 & 1026 & $21.2 \%(20.0-22.3)$ & Reference & Reference \\
\hline 2007-2009 & 6104 & 1013 & $16.6 \%(15.7-17.5)$ & $0.74(0.67-0.82)$ & $0.74(0.67-0.82)$ \\
\hline 2010-2012 & 5547 & 950 & $17.1 \%(16.1-18.1)$ & $0.77(0.70-0.85)$ & $0.78(0.71-0.86)$ \\
\hline $2013-2016$ & 7042 & 1115 & $15.8 \%(15.0-16.7)$ & $0.70(0.64-0.77)$ & $0.71(0.64-0.78)$ \\
\hline
\end{tabular}

*Adjusted for sex, age, smoking, hypertension, previous ischemic stroke, peripheral artery disease, statin treatment, antiplatelet treatment, oral anti-coagulant treatment

treatment patterns, diabetes patients with chronic coronary syndrome remain at higher risk of MACE than patients without diabetes. An intensive, multifactorial treatment strategy before the development of cardiovascular disease is essential in order to minimize cardiovascular risk among diabetes patients.

\section{Abbreviations}

MI: Myocardial infarction; CAD: Coronary artery disease; PCI: Percutaneous coronary intervention; FFR: Fractional flow reserve; CAG: Coronary angiography; CABG: Coronary artery bypass grafting; BMI: Body mass index; ADP: Adenosine diphosphate; ACE: Angiotensin-converting enzyme; ARB: Angiotensin II receptor blocker; MACE: Major adverse cardiovascular event; ATC: Anatomic Therapeutic Chemical; ICD-10: International Classification of Diseases 10; IRR: Incidence rate ratio; alRR: Adjusted incidence rate ratio; DES: Drug-eluting stent.

\section{Supplementary Information}

The online version contains supplementary material available at https://doi. org/10.1186/s12872-021-02312-y.

Additional file 1: Table S1 and Table S2 show changes in statin and ADP inhibitor treatment in diabetes and non-diabetes patients from 2004 to 2016. Table S3 compares two-year risks of major adverse cardiovascular events between diabetes and non-diabetes patients from 2004 to 2016 .
Table S4 and S5 show two-year risks of major adverse cardiovascular events in diabetes and non-diabetes patients with chronic coronary disease and obstructive coronary disease. Table S6 and S7 show two-year risks of major adverse cardiovascular events in diabetes and non-diabetes patients stratified by sex. Table S8 and S9 show two-year risks of major adverse cardiovascular events in diabetes and non-diabetes patients stratified by age above or below 70 years. Table S10 shows two-year risks of coronary revascularization after coronary angiography in diabetes and non-diabetes patients with any coronary artery disease and in diabetes and non-diabetes patients with obstructive coronary artery disease.

\section{Acknowledgements}

Not applicable.

\section{Authors' contributions}

ESJ, KKWO, CG, PGT, LOJ, BR, PLP, RWT, and MM conceptualized the study. ESJ, KKWO, and MM designed the study. KKWO was responsible for data acquisition, data management and analysis. ESJ, KKWO, CG, PGT, LOJ, BR, PLP, RWT, and MM contributed to data interpretation. ESJ wrote the first draft of the manuscript. EJS, KKWO, CG, PGT, LOJ, BR, PLP, RWT, and MM provided significant revision of the manuscript and gave final approval for publication. All authors read and approved the final manuscript.

\section{Funding}

This study was funded by the Department of Cardiology, Aarhus University Hospital, Aarhus, Denmark.

\section{Availability of data and materials}

According to Danish data protection regulations, data cannot be made publicly available. 


\section{Declarations}

\section{Ethics approval and consent to participate}

This study was approved by the Danish Data Protection Agency (record no. 1-16-02-193-18). According to Danish regulations, observational non-interventional registry-based studies do not require approval from ethics committees or informed consent from participants.

\section{Consent for publication}

Informed consent is not required from participants in registry-based, noninterventional cohort studies according to Danish regulation.

\section{Competing interests}

The authors declare that they have no competing interests. Department of Clinical Epidemiology is, however, involved in studies with funding from various companies as research grants to and administered by Aarhus University. None of these studies are related to the current study. Dr. Maeng has received advisory board fees from Boehringer-Ingelheim, AstraZeneca, Novo Nordisk, BMS, Boston Scientific, and Bayer.

\section{Author details}

'Department of Cardiology, Aarhus University Hospital, Palle Juul-Jensens Boulevard 99, Aarhus N, Denmark. ${ }^{2}$ Department of Cardiology, Odense University Hospital, Odense, Denmark. ${ }^{3}$ Department of Cardiology, Aalborg University Hospital, Aalborg, Denmark. ${ }^{4}$ Steno Diabetes Center Aarhus, Aarhus University Hospital, Aarhus, Denmark. ${ }^{5}$ Department of Clinical Epidemiology, Aarhus University Hospital, Aarhus, Denmark.

Received: 21 June 2021 Accepted: 8 October 2021

Published online: 04 December 2021

\section{References}

1. Gaede $\mathrm{P}$, Lund-Andersen $\mathrm{H}$, Parving $\mathrm{HH}$, Pedersen O. Effect of a multifactorial intervention on mortality in type 2 diabetes. N Engl J Med. 2008;358:580-91.

2. Cosentino F, Grant PJ, Aboyans V, et al. 2019 ESC Guidelines on diabetes, pre-diabetes, and cardiovascular diseases developed in collaboration with the EASD. Eur Heart J. 2020;41:255-323.

3. Disease C, Management R. Standards of medical care in diabetes-2020. Diabetes Care. 2020;43(Supplement 1):S111-34.

4. Effect of intensive diabetes management on macrovascular events and risk factors in the Diabetes Control and Complications Trial. Am J Cardio 1995;75:894-903.

5. Tight blood pressure control and risk of macrovascular and microvascular complications in type 2 diabetes: UKPDS 38. UK Prospective Diabetes Study Group. BMJ 1998;317:703-13.

6. Colhoun HM, Betteridge DJ, Durrington PN, et al. Primary prevention of cardiovascular disease with atorvastatin in type 2 diabetes in the Collaborative Atorvastatin Diabetes Study (CARDS): multicentre randomised placebo-controlled trial. Lancet. 2004;364:685-96.

7. Gyldenkerne C, Knudsen JS, Olesen KKW et al. Nationwide trends in cardiac risk and mortality in patients with incident type 2 diabetes: a Danish cohort study. Diabetes Care. 2021

8. Pijls NH, Fearon WF, Tonino PA, et al. Fractional flow reserve versus angiography for guiding percutaneous coronary intervention in patients with multivessel coronary artery disease: 2-year follow-up of the FAME (Fractional Flow Reserve Versus Angiography for Multivessel Evaluation) study. J Am Coll Cardiol. 2010;56:177-84.

9. Maeng $\mathrm{M}$, Tilsted $\mathrm{HH}$, Jensen $\mathrm{LO}$, et al. Differential clinical outcomes after 1 year versus 5 years in a randomised comparison of zotarolimuseluting and sirolimus-eluting coronary stents (the SORT OUT III study): a multicentre, open-label, randomised superiority trial. Lancet. 2014;383:2047-56.

10. Farkouh ME, Domanski $M$, Sleeper $L A$, et al. Strategies for multivessel revascularization in patients with diabetes. N Engl J Med. 2012:367:2375-84.
11. Schmidt M, Maeng M, Madsen M, Sorensen HT, Jensen LO, Jakobsen CJ. The Western Denmark heart registry: its influence on cardiovascular patient care. J Am Coll Cardiol. 2018;71:1259-72

12. Pottegård A, Schmidt SAJ, Wallach-Kildemoes H, Sørensen HT, Hallas J, Schmidt M. Data resource profile: the Danish national prescription registry. Int J Epidemiol. 2017;46:798-798f.

13. Schmidt M, Schmidt SA, Sandegaard JL, Ehrenstein V, Pedersen L, Sorensen HT. The Danish National Patient Registry: a review of content, data quality, and research potential. Clin Epidemiol. 2015;7:449-90.

14. Helweg-Larsen K. The Danish register of causes of death. Scand J Public Health. 2011;39:26-9.

15. Schmidt M, Pedersen L, Sorensen HT. The Danish civil registration system as a tool in epidemiology. Eur J Epidemiol. 2014;29:541-9.

16. Charlson ME, Pompei P, Ales KL, MacKenzie CR. A new method of classifying prognostic comorbidity in longitudinal studies: development and validation. J Chronic Dis. 1987:40:373-83.

17. Egholm G, Madsen M, Thim T, et al. Evaluation of algorithms for registrybased detection of acute myocardial infarction following percutaneous coronary intervention. Clin Epidemiol. 2016;8:415-23.

18. Krarup LH, Boysen G, Janjua H, Prescott E, Truelsen T. Validity of stroke diagnoses in a National Register of Patients. Neuroepidemiology. 2007;28:150-4.

19. Gyldenkerne C, Olesen KK, Thrane PG, et al. Diabetes is not a risk factor for myocardial infarction in patients without coronary artery disease: a study from the Western Denmark Heart Registry. Diab Vasc Dis Res. 2020;17:1479164120941809.

20. Zou G. A modified Poisson regression approach to prospective studies with binary data. Am J Epidemiol. 2004;159:702-6.

21. Steensig K, Olesen KKW, Madsen M, et al. A novel model for prediction of thromboembolic and cardiovascular events in patients without atrial fibrillation. Am J Cardiol. 2020;131:40-8.

22. Farnier M, Portal JJ, Maigret P. Efficacy of atorvastatin compared with simvastatin in patients with hypercholesterolemia. J Cardiovasc Pharmacol Ther. 2000:5:27-32

23. Head SJ, Milojevic M, Daemen J, et al. Mortality after coronary artery bypass grafting versus percutaneous coronary intervention with stenting for coronary artery disease: a pooled analysis of individual patient data. Lancet. 2018;391:939-48.

24. Madhavan MV, Redfors B, Ali ZA, et al. Long-term outcomes after revascularization for stable ischemic heart disease: an individual patient-leve pooled analysis of 19 randomized coronary stent trials. Circ Cardiovasc Interv. 2020;13:e008565

25. Olesen KKW, Pareek M, Madsen M, et al. Ten-year outcomes of sirolimuseluting versus zotarolimus-eluting coronary stents in patients with versus without diabetes mellitus (SORT OUT III). Am J Cardiol. 2020;125:349-53.

26. Bavishi C, Baber U, Panwar S, et al. Efficacy and safety of everolimus and zotarolimus-eluting stents versus first-generation drug-eluting stents in patients with diabetes: a meta-analysis of randomized trials. Int J Cardiol. 2017;230:310-8.

27. Borren NM, Ottervanger JP, Reinders MA, Kedhi E. Coronary artery stenoses more often overestimated in older patients: angiographic stenosis overestimation in elderly. Int J Cardiol. 2017;241:46-9.

28. Gyldenkerne C, Knudsen JS, Olesen KKW et al. Nationwide trends for myocardial infarction and mortality among patients with type 2 diabetes mellitus. Presented as late-breaking clinical science at the European society of cardiology. 2020.

29. Rawshani A, Rawshani A, Franzen S, et al. Mortality and cardiovascular disease in type 1 and type 2 diabetes. N Engl J Med. 2017;376:1407-18.

30. Szummer K, Wallentin L, Lindhagen L, et al. Improved outcomes in patients with ST-elevation myocardial infarction during the last 20 years are related to implementation of evidence-based treatments: experiences from the SWEDEHEART registry 1995-2014. Eur Heart J. 2017:38:3056-65.

31. Szummer K, Wallentin L, Lindhagen $L$, et al. Relations between implementation of new treatments and improved outcomes in patients with nonST-elevation myocardial infarction during the last 20 years: experiences from SWEDEHEART registry 1995 to 2014. Eur Heart J. 2018:39:3766-76.

32. Thygesen K, Alpert JS, White HD, et al. Universal definition of myocardial infarction. Circulation. 2007:116:2634-53.

33. Thygesen K, Alpert JS, Jaffe AS, et al. Third universal definition of myocardial infarction. Nat Rev Cardiol. 2012;9:620-33.

34. Odqvist M, Andersson PO, Tygesen H, Eggers KM, Holzmann MJ. Highsensitivity troponins and outcomes after myocardial infarction. J Am Coll Cardiol. 2018;71:2616-24. 
35. Sardu C, Paolisso P, Sacra C, et al. Effects of metformin therapy on coronary endothelial dysfunction in patients with prediabetes with stable angina and nonobstructive coronary artery stenosis: the CODYCE multicenter prospective study. Diabetes Care. 2019;42:1946-55.

36. Sardu C, D'Onofrio N, Torella M, et al. Pericoronary fat inflammation and Major Adverse Cardiac Events (MACE) in prediabetic patients with acute myocardial infarction: effects of metformin. Cardiovasc Diabetol. 2019:18:126.

\section{Publisher's Note}

Springer Nature remains neutral with regard to jurisdictional claims in published maps and institutional affiliations.

- fast, convenient online submission

- thorough peer review by experienced researchers in your field

- rapid publication on acceptance

- support for research data, including large and complex data types

- gold Open Access which fosters wider collaboration and increased citations

- maximum visibility for your research: over $100 \mathrm{M}$ website views per year

At $\mathrm{BMC}$, research is always in progress.

Learn more biomedcentral.com/submissions 